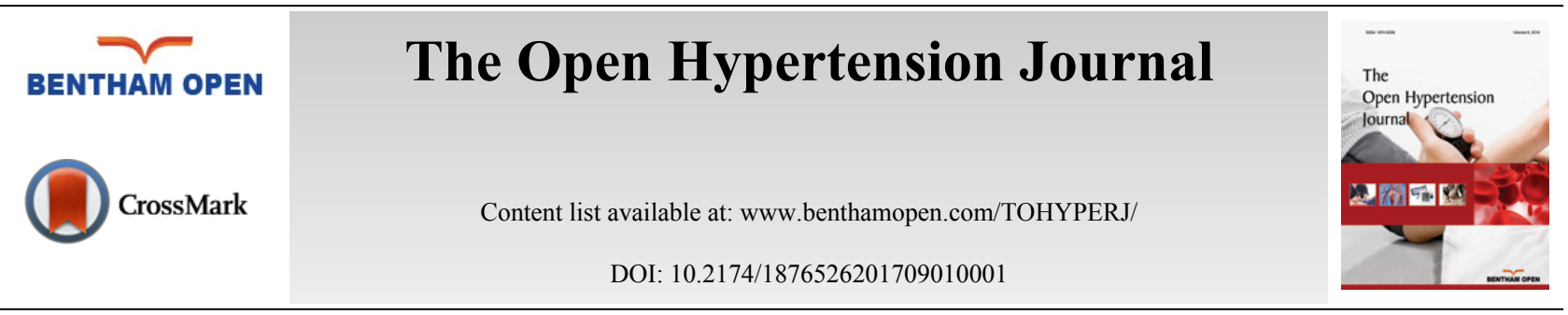

\title{
EDITORIAL
}

\section{Arterial Stiffness and Nonalcoholic Fatty Liver Disease: Which is the Chicken and Which is the Egg?}

Nonalcoholic fatty liver disease (NAFLD) is diagnosed when there is an accumulation of fat in the liver $(>5 \%)$ in the absence of alcohol abuse or other chronic liver diseases (CLD) [1]. It is the most frequent cause of elevated liver enzyme levels worldwide [2]. NAFLD can progress to nonalcoholic steatohepatitis (NASH) with liver inflammation, fibrosis, cirrhosis or finally to hepatocellular cancer (HCC). However, cardiovascular disease (CVD) is the most frequent cause of death or disability in NASH patients [3].

NAFLD has a median prevalence of $20 \%$ worldwide [4] and around 25\% in US and Europe [4] and 9 to $45 \%$ in Southeast Asia [5, 6]. NAFLD, but mainly its advanced form, NASH, complicated with liver inflammation with or without liver fibrosis, are independent and major CVD risk factors, with NAFLD patients dying from CVD rather than liver-related complications (cirrhosis or HCC) [1, 2].

When the left ventricle of the heart contracts, a pulse wave which travels through the aorta and the arterial system is generated [7]. The speed of travel of this pulse wave (pulse wave velocity [PWV]) is related to the compliance of the arteries [7]. Arterial stiffness (AS) emerges when the elastic properties of large arteries are diminished [7]. In other words, arterial stiffness occurs as a consequence of biological aging and arteriosclerosis [7, 8]. Several degenerative changes that occur with age in the walls of large elastic arteries are thought to contribute to increased stiffening over time, including the mechanical fraying of lamellar elastin structures within the wall due to mechanical stress, changes in the structure and increases in the content of arterial collagen proteins, partially as a compensatory mechanism against the loss of arterial elastin and partially due to fibrosis [7,8]. Arterial stiffness is an independent and major CVD risk factor, is directly related to the overall atherosclerotic burden and the incidence of CVD events [8]. The co-existence of NAFLD/NASH with AS dramatically increases the CVD risk.

The possible biological mechanisms linking NAFLD/NASH with increased AS are mostly unknown and it is also unknown whether they are parallel or common [9], but it is possible that common pathways involving chronic lowgrade inflammation and adipokine imbalance are involved [10]. An early study reported that NAFLD is related to AS (n $=213$ ), especially in women [11]. The study concluded that there is an association between serum adiponectin concentration, PWV and NAFLD [11]. Since then a lot of progress has been made.

Initially, it was believed that AS is the cause of NAFLD. The relationship between NAFLD and AS was investigated in a large cohort $(n=4,860)$ from Taiwan. The results showed that AS was increased only in subjects with normal glucose levels [12]. Thus, people with prediabetes and those with type 2 diabetes mellitus (T2DM), in whom the prevalence of NAFLD is approximately $90 \%$ [2], did not have AS [12]. The results of this study were reinforced by another large study in 1,296 Chinese subjects [13], which suggested that NAFLD is prevalent and related with AS in normotensive, non-diabetic young and middle-aged subjects [13]. This means that there was increased AS related to NAFLD in the absence of major NAFLD risk factors, suggesting that AS is the major pathogenetic mechanism of NAFLD/NASH [13].

Results from the Rio-T2DM cohort study $(\mathrm{n}=291)$ suggest that the presence of AS contributes substantially to liver fibrosis [14]. NAFLD in its initial form of simple steatosis is a rather benign situation without excess mortality, while NASH with advanced fibrosis increases total mortality by $70 \%$, due to an increase in CVD mortality by nearly $300 \%$ [2]. Thus, in this cohort, AS induced a substantial deterioration of pre-existing NASH and this had an immediate impact on outcomes. In a study from Turkey, it was shown that pentraxin 3 (PTX3) levels, a marker of inflammation which is produced by the arterial wall, were higher in NAFLD patients with fibrosis than in NAFLD patients without fibrosis and 
healthy subjects, independently of metabolic syndrome (MetS) components, while PTX3 levels were strongly correlated with AS in patients with NAFLD [15]. In any case, the fact that AS is the chicken and not the egg has been shown in specific (non western) ethnic groups.

On the contrary, it was reported in a very large study $(8,603$ subjects [6, 662 males]) that NAFLD/NASH is strongly related with AS, particularly in females, regardless of the degree of metabolic abnormalities and thus NAFLD may be a useful predictor of early arteriosclerosis (increased AS) [16]. In another study, it was shown in adolescents that the increase of age, liver fat, and triglyceride levels are closely related to increased AS in adults [17]. Furthermore, in a study from China that included 2,550 middle aged participants with ultrasound confirmed NAFLD, it was shown that in patients with NAFLD/NASH, advanced liver fibrosis was related to carotid intima-media thickness (cITM), a surrogate of coronary atherosclerosis, and AS, regardless of the presence of conventional cardiometabolic risk factors or insulin resistance (IR) [18]. A very large study in middle-aged and elderly Chinese with NAFLD $(n=8,632)$ reported similar findings [19]. In a study in Asian Indians, NAFLD/NASH was shown to be associated with subclinical atherosclerosis, AS, and endothelial dysfunction independently of obesity and MetS [20]. Therefore, the CVD risk of NAFLD/NASH may extend far beyond that of MetS.

In a study from Greece, NAFLD/NASH was reported to be related with AS and endothelial dysfunction [21]. Since these arterial indices are independent CVD risk factors, these findings have important implications for CVD risk prevention in patients with both NAFLD/NASH and AS.

The above studies suggest that NAFLD/NASH is the chicken and AS is the egg. Nevertheless, it is also possible that NAFLD/NASH and AS might progress in parallel, since they have common risk factors [9] and exacerbate one another in a vicious cycle, leading to a geometrical increase in CVD events. This underlines the fact that we should urgently treat them both to reduce CVD morbidity and mortality.

There is evidence that both conditions could be treated by the same drug (the potent statins atorvastatin and rosuvastatin), increasing the compliance of the patients to the treatment. This reduces the interest about which caused the other or if they both result from common risk factors.

The use of atorvastatin was shown to ameliorate NAFLD/NASH in 3 post hoc analyses of prospective survival studies. In the Greek Atorvastatin and Coronary Heart Disease Evaluation (GREACE) Study $(n=1,600)$ [22], the Assessing the Treatment Effect in Metabolic Syndrome Without Perceptible Diabetes (ATTEMPT) study $(\mathrm{n}=1,123)[23]$ and the Incremental Decrease in End Points Through Aggressive Lipid Lowering (IDEAL) trial (n=8,863) [24], atorvastatin in doses 20-80 mg/d safely ameliorated NAFLD/NASH and most importantly halved CVD morbidity and mortality in comparison with those that were on a statin and had normal liver function tests [22 - 24]. In 2 multicentre prospective studies ( $n=107$ and $n=346)$ with statins, including atorvastatin, it was shown that there was a reduction in necroinflammation, serious fibrosis and other NAFLD/NASH characteristics $[25,26]$. On the other hand, atorvastatin has been shown to reduce arterial stiffness without affecting blood pressure [27]. However, this is not a class effect (as in the case of NAFLD/NASH [28]) and not all statins reduce aortic stiffness (only atorvastatin or rosuvastatin significantly ameliorated AS) $[29,30]$. Thus, only atorvastatin and rosuvastatin can treat both NAFLD/NASH and AS and substantially reduce CVD risk [30, 31].

Recent (unpublished) data from our clinic suggest that there is a strong correlation between AS and liver fibrosis (but not with simple steatosis), and that rosuvastatin in low and high doses substantially improves both, reducing CVD risk. Given that several hundred of millions of people suffer from NAFLD/NASH $[1,2]$ and a similar number from AS, the use of atorvastatin or rosuvastatin in these people is a very useful and practical tool if we take into consideration the fact that there is no generally accepted treatment for NAFLD/NASH, or any other drug improving both NAFLD/NASH and AS.

\section{DECLARATION OF INTEREST}

This Editorial was written independently; no company or institution supported the authors financially or by providing a professional writer. Some of the authors have given talks, attended conferences and participated in trials and advisory boards sponsored by various pharmaceutical companies. There is no specific conflict of interest in regard to the specific Editorial. 


\section{REFERENCES}

[1] European Association for the Study of the Liver (EASL); European Association for the Study of Diabetes (EASD); European Association for the Study of Obesity (EASO). EASL-EASD-EASO Clinical Practice Guidelines for the management of non-alcoholic fatty liver disease. J Hepatol 2016; 64(6): 1388-402. [http://dx.doi.org/10.1016/j.jhep.2015.11.004] [PMID: 27062661]

[2] Athyros VG, Tziomalos K, Katsiki N, Doumas M, Karagiannis A, Mikhailidis DP. Cardiovascular risk across the histological spectrum and the clinical manifestations of non-alcoholic fatty liver disease: An update. World J Gastroenterol 2015; 21(22): 6820-34. [PMID: 26078558]

[3] Aronow WS. Nonalcoholic fatty liver disease is associated with coronary artery disease and subclinical atherosclerosis. Future Cardiol 2016; 12(4): 393-6. [http://dx.doi.org/10.2217/fca-2016-0033] [PMID: 27291253]

[4] Bellentani S. The epidemiology of non-alcoholic fatty liver disease. Liver Int 2017; 37(Suppl. 1): 81-4. [http://dx.doi.org/10.1111/liv.13299] [PMID: 28052624]

[5] Farrell GC, Wong VW, Chitturi S. NAFLD in Asiaas common and important as in the West. Nat Rev Gastroenterol Hepatol 2013; 10(5): 307-18. [http://dx.doi.org/10.1038/nrgastro.2013.34] [PMID: 23458891]

[6] Mohan V, Farooq S, Deepa M, Ravikumar R, Pitchumoni CS. Prevalence of non-alcoholic fatty liver disease in urban south Indians in relation to different grades of glucose intolerance and metabolic syndrome. Diabetes Res Clin Pract 2009; 84(1): 84-91. [http://dx.doi.org/10.1016/j.diabres.2008.11.039] [PMID: 19168251]

[7] Díez J. Arterial stiffness and extracellular matrix. Adv Cardiol 2007; 44: 76-95. [http://dx.doi.org/10.1159/000096722] [PMID: 17075200]

[8] Palombo C, Kozakova M. Arterial stiffness, atherosclerosis and cardiovascular risk: Pathophysiologic mechanisms and emerging clinical indications. Vascul Pharmacol 2016; 77: 1-7.

[http://dx.doi.org/10.1016/j.vph.2015.11.083] [PMID: 26643779]

[9] Villela-Nogueira CA, Leite NC, Cardoso CR, Salles GF. NAFLD and Increased Aortic Stiffness: Parallel or Common Physiopathological Mechanisms? Int J Mol Sci 2016; 17(4): E460. [http://dx.doi.org/10.3390/ijms17040460] [PMID: 27104526]

[10] Jain S, Khera R, Corrales-Medina VF, Townsend RR, Chirinos JA. Inflammation and arterial stiffness in humans. Atherosclerosis 2014; 237(2): 381-90.

[http://dx.doi.org/10.1016/j.atherosclerosis.2014.09.011] [PMID: 25463062]

[11] Kim SG, Kim HY, Seo JA, et al. Relationship between serum adiponectin concentration, pulse wave velocity and nonalcoholic fatty liver disease. Eur J Endocrinol 2005; 152(2): 225-31.

[http://dx.doi.org/10.1530/eje.1.01842] [PMID: 15745930]

[12] Chou CY, Yang YC, Wu JS, Sun ZJ, Lu FH, Chang CJ. Non-alcoholic fatty liver disease associated with increased arterial stiffness in subjects with normal glucose tolerance, but not pre-diabetes and diabetes. Diab Vasc Dis Res 2015; 12(5): 359-65. [http://dx.doi.org/10.1177/1479164115585009] [PMID: 26008803]

[13] Yu XY, Zhao Y, Song XX, Song ZY. Association between non-alcoholic fatty liver disease and arterial stiffness in the non-obese, nonhypertensive, and non-diabetic young and middle-aged Chinese population. J Zhejiang Univ Sci B 2014; $15(10)$ : 879-87. [http://dx.doi.org/10.1631/jzus.B1400028] [PMID: 25294377]

[14] Leite NC, Villela-Nogueira CA, Ferreira MT, Cardoso CR, Salles GF. Increasing aortic stiffness is predictive of advanced liver fibrosis in patients with type 2 diabetes: the Rio-T2DM cohort study. Liver Int 2016; 36(7): 977-85. [http://dx.doi.org/10.1111/liv.12994] [PMID: 26509555]

[15] Ozturk K, Kurt O, Dogan T, et al. Pentraxin 3 Is a Predictor for Fibrosis and Arterial Stiffness in Patients with Nonalcoholic Fatty Liver Disease. Gastroenterol Res Pract 2016. 2016: 1417962.

[16] Zhu WH, Fang LZ, Lu CR, et al. Correlation between non-alcoholic fatty liver with metabolic risk factors and brachial-ankle pulse wave velocity. World J Gastroenterol 2015; 21(35): 10192-9. [PMID: 26401084]

[17] Rider OJ, Banerjee R, Rayner JJ, et al. Investigating a Liver Fat: Arterial Stiffening Pathway in Adult and Childhood Obesity. Arterioscler Thromb Vasc Biol 2016; 36(1): 198-203. [PMID: 26564820]

[18] Chen Y, Xu M, Wang T, et al. Advanced fibrosis associates with atherosclerosis in subjects with nonalcoholic fatty liver disease Atherosclerosis 2015; 241(1): 145-50. [http://dx.doi.org/10.1016/j.atherosclerosis.2015.05.002] [PMID: 25988358]

[19] Huang Y, Bi Y, Xu M, et al. Nonalcoholic fatty liver disease is associated with atherosclerosis in middle-aged and elderly Chinese. Arterioscler Thromb Vasc Biol 2012; 32(9): 2321-6. [http://dx.doi.org/10.1161/ATVBAHA.112.252957] [PMID: 22814750] 
[20] Thakur ML, Sharma S, Kumar A, et al. Nonalcoholic fatty liver disease is associated with subclinical atherosclerosis independent of obesity and metabolic syndrome in Asian Indians. Atherosclerosis 2012; 223(2): 507-11. [http://dx.doi.org/10.1016/j.atherosclerosis.2012.06.005] [PMID: 22748277]

[21] Vlachopoulos C, Manesis E, Baou K, et al. Increased arterial stiffness and impaired endothelial function in nonalcoholic Fatty liver disease: a pilot study. Am J Hypertens 2010; 23(11): 1183-9. [http://dx.doi.org/10.1038/ajh.2010.144] [PMID: 20634799]

[22] Athyros VG, Tziomalos K, Gossios TD, et al. Safety and efficacy of long-term statin treatment for cardiovascular events in patients with coronary heart disease and abnormal liver tests in the Greek Atorvastatin and Coronary Heart Disease Evaluation (GREACE) Study: a posthoc analysis. Lancet 2010; 376(9756): 1916-22. [http://dx.doi.org/10.1016/S0140-6736(10)61272-X] [PMID: 21109302]

[23] Athyros VG, Elisaf MS, Alexandrides T, et al. Long-term impact of multifactorial treatment on new-onset diabetes and related cardiovascular events in metabolic syndrome: a post hoc ATTEMPT analysis. Angiology 2012; 63(5): 358-66. [http://dx.doi.org/10.1177/0003319711421341] [PMID: 22007026]

[24] Tikkanen MJ, Fayyad R, Faergeman O, et al. Effect of intensive lipid lowering with atorvastatin on cardiovascular outcomes in coronary heart disease patients with mild-to-moderate baseline elevations in alanine aminotransferase levels. Int J Cardiol 2013 ; $168(4)$ : $3846-52$. [http://dx.doi.org/10.1016/j.ijcard.2013.06.024] [PMID: 24001698]

[25] Dongiovanni P, Petta S, Mannisto V, et al. Statin use and non-alcoholic steatohepatitis in at risk individuals. J Hepatol 2015; 63(3): 705-12. [http://dx.doi.org/10.1016/j.jhep.2015.05.006] [PMID: 25980762]

[26] Nascimbeni F, Aron-Wisnewsky J, Pais R, et al. Statins, antidiabetic medications and liver histology in patients with diabetes with nonalcoholic fatty liver disease. BMJ Open Gastroenterol 2016; 3(1): e000075. [http://dx.doi.org/10.1136/bmjgast-2015-000075] [PMID: 27110380]

[27] Kontopoulos AG, Athyros VG, Pehlivanidis AN, Demitriadis DS, Papageorgiou AA, Boudoulas H. Long-term treatment effect of atorvastatin on aortic stiffness in hypercholesterolaemic patients. Curr Med Res Opin 2003; 19(1): 22-7. [http://dx.doi.org/10.1185/030079902125001290] [PMID: 12661776]

[28] Eslami L, Merat S, Malekzadeh R, Nasseri-Moghaddam S, Aramin H. Statins for non-alcoholic fatty liver disease and non-alcoholic steatohepatitis. Cochrane Database Syst Rev 2013; (12): CD008623. [PMID: 24374462]

[29] Shige H, Dart A, Nestel P. Simvastatin improves arterial compliance in the lower limb but not in the aorta. Atherosclerosis 2001; 155(1): 245-50. [http://dx.doi.org/10.1016/S0021-9150(00)00558-X] [PMID: 11223448]

[30] Toyama K, Sugiyama S, Oka H, et al. Combination treatment of rosuvastatin or atorvastatin, with regular exercise improves arterial wall stiffness in patients with coronary artery disease. PLoS One 2012; 7(7): e41369. [http://dx.doi.org/10.1371/journal.pone.0041369] [PMID: 22829944]

[31] Kargiotis K, Athyros VG, Giouleme O, et al. Resolution of non-alcoholic steatohepatitis by rosuvastatin monotherapy in patients with metabolic syndrome. World J Gastroenterol 2015; 21(25): 7860-8. [http://dx.doi.org/10.3748/wjg.v21.i25.7860] [PMID: 26167086]

Theodora Griva

Second Propedeutic Department of Internal Medicine Medical School, Aristotle University of Thessaloniki Hippocration Hospital Thessaloniki, Greece

Chrysoula Boutari

Second Propedeutic Department of Internal Medicine Medical School, Aristotle University of Thessaloniki Hippocration Hospital Thessaloniki, Greece

Konstantinos Tziomalos

First Propedeutic Department of Internal Medicine Medical School, Aristotle University of Thessaloniki AHEPA Hospital Thessaloniki, Greece 
Michael Doumas

Second Propedeutic Department of Internal Medicine Medical School, Aristotle University of Thessaloniki

Hippocration Hospital

Thessaloniki, Greece

Asterios Karagiannis

Second Propedeutic Department of Internal Medicine Medical School, Aristotle University of Thessaloniki

Hippocration Hospital Thessaloniki, Greece

Vasilios G. Athyros

Head of Atherosclerosis and Metabolic Disease Units Second Propedeutic Department of Internal Medicine Medical School

Aristotle University of Thessaloniki

Thessaloniki, 55 132, Greece

E-mail: vathyros@gmail.com

E-mail: athyros@med.auth.gr

\section{(c) Griva et al.}

This is an open access article distributed under the terms of the Creative Commons Attribution 4.0 International Public License (CC-BY 4.0), a copy of which is available at: https://creativecommons.org/licenses/by/4.0/legalcode. This license permits unrestricted use, distribution, and reproduction in any medium, provided the original author and source are credited. 